\title{
Meaning Reconstruction as an Approach to Analyze Critical Dimensions of $\mathrm{HCl}$ Research
}

Colin M. Gray

Purdue University

401 N. Grant Street, Room 355

West Lafayette, IN 47907, USA

gray42@purdue.edu

Austin L. Toombs

Indiana University

901 E. 10th Street

Bloomington, IN 47408, USA

altoombs@indiana.edu

\section{Christian McKay}

Indiana University

420 N. Walnut Street, Room 114

Bloomington, IN 47404, USA

mckayc@indiana.edu

Permission to make digital or hard copies of all or part of this work for personal or classroom use is granted without fee provided that copies are not made or distributed for profit or commercial advantage and that copies bear this notice and the full citation on the first page. Copyrights for components of this work owned by others than the author(s) must be honored. Abstracting with credit is permitted. To copy otherwise, or republish, to post on servers or to redistribute to lists, requires prior specific permission and/or a fee. Request permissions from Permissions@acm.org.

CHI'16 Extended Abstracts, May 07 - 12, 2016, San Jose, CA, USA Copyright is held by the owner/author(s). Publication rights licensed to ACM. ACM 978-1-4503-4082-3/16/05 ..\$15.00

DOI: http://dx.doi.org/10.1145/2851581.2892571

\begin{abstract}
A critical tradition has taken hold in $\mathrm{HCI}$, yet research methods needed to meaningfully engage with critical questions in the qualitative tradition are nascent. In this paper, we explore one critical qualitative research approach that allows researchers to probe deeply into the relationships between communicative acts and social structures. Meaning reconstruction methods are described and illustrated using examples from HCI research, demonstrating how social norms can be traced as they are claimed and reproduced. We conclude with implications for strengthening rigorous critical inquiry in $\mathrm{HCI}$ research, including the use of extant critical research methods to document transparency and thick description.
\end{abstract}

\section{Author Keywords}

Critical theory; reconstructive analysis; rigor.

\section{ACM Classification Keywords}

H.5.m. Information interfaces and presentation (e.g., HCI): Miscellaneous.

\section{Introduction}

The research landscape of $\mathrm{HCI}$ is increasingly shaped by the ways in which social norms, ethics, and values 
are implicated in technologically-mediated interactions. However, the shift from a technologically-centered research paradigm to one that is marked by criticality, sociality, and power has been complicated. Criticality in an HCI context has most frequently been couched in issues of feminism [1], reflection on social systems and normative commitments $[12,19,23]$, and the ethical responsibility of designing technologies $[13,21,22]$. Whereas technocentric research characteristic of first and second paradigm HCI [10] generally operated in an experimental scientific mode, using measures that were easily quantified and compared, research methods in contemporary $\mathrm{HCI}$ research have been more diffuse, drawing on numerous social science and humanist traditions $[3,16]$. In a traditional scientific or "scientistic" mode, reflection on the epistemological stance of the researcher and her method was uneven and infrequent. In contrast, methodological approaches originating across multiple disciplines require consideration and articulation of epistemological stances in order to communicate the variety of paths towards validity and rigor. While we find inherent value in all of these methodological approaches and the traditions from which they are derived, our goal in this paper is to explore methodological rigor when engaging in critical qualitative analysis in an HCI context.

Numerous qualitative methodologies and fields have been drawn upon from social science for use in an $\mathrm{HCI}$ context-such as grounded theory, thematic analysis, and discourse analysis. However, to incorporate the critical dimension as a key component of our methodology, richer methods are needed to comprehensively document and explain the ways in which communicative acts and social norms are linked, in both temporal and experiential ways. Rigorous qualitative research in the naturalistic tradition relies upon thick description, researcher transparency, disclosure, reflexivity, and sufficient explanation of method to allow an external entity access into the mindset of the researcher $[9,11]$. In this regard current critical research-if not most qualitative research-in the HCI community is often a "black box," lacking clear description of how critical inferences are drawn from data in a rigorous way. For instance, recent discussions on the ways in which critical designs might be defined and interpreted $[5,18]$ have opened the question of critical discourse beyond the context of research through design to the broader qualitative research tradition in HCI. Such research has often left the mindset of interpretation or derivation of critical implications undefined or undertheorized, resulting in a poor representation of the researcher's reflexive role and transparency of collection and analysis.

In this paper, we will demonstrate one rigorous methodological approach comprised of multiple integrated methods that allows reflection on topics of interest in the $\mathrm{HCI}$ community, using a metatheoretica toolkit and perspective from the critical qualitative inquiry tradition. Within the context of third-wave critical theory, Phil Carspecken $[6,7,8]$ has been a dominant voice, drawing together Jürgen Habermas' Theory of Communicative Action (TCA), Anthony Giddens' understanding of structuration and the reproduction of social systems, and J. L. Austin's Speech Act Theory and understanding of the illocutionary content of speech into a comprehensive methodological approach.

The authors have used this metatheoretical toolkit in the context of design education $[14,15]$ and maker 


\section{Objective Claims}

Multiple access perspective, whereby a phenomenon may be judged for what it is or what appears by multiple subjects. Resolved through mutually agreed upon standards of truth and efficacy, and are concerned with "the" world.

\section{Subjective Claims}

Limited access perspective

for those outside the

subject's experience.

Resolved through adequacy

of standards for criticism, and are concerned with "my" world.

Normative-Evaluative Claims What should or ought to be. Resolved by countering the rightness of norms or actions, and is concerned with "our" world. practices [23] to document critical dimensions of identity formation in relation to technology and design practices, and the ways in which communication in education and maker settings reproduce and enable the broader social system. In the following sections, we will outline key terminology that explains a Habermasian approach to communicative acts and social systems. Our intention is to build a shared understanding of the interplay between epistemological understandings of the nature of communication and the methods used to reconstruct and thereby analyze communicative acts. It may be helpful for the reader to work through the two examples provided in section three alongside the explanation of Habermas' three formal worlds.

\section{Habermas' Three Formal Worlds}

In his Theory of Communicative Action, Habermas proposes that all communicative acts have three dimensions, related to "formal worlds" [6]. Each of these worlds takes on a different subject position or perspective, and can be judged by differing standards of validity (i.e., what makes an act internally rational) related to the world the claim resides within. All communicative acts contain validity claims in all three formal worlds (discussed below in Intersubjective Space), which fuse together in a moment of mutuality where we understand ourselves, and others have the capability to understand us. These worlds are formal and not just simple ontological worlds because any communicative act will necessarily claim or represent the boundaries between these worlds in contestable ways, while also recognizing there is no way to access the formal worlds without addressing the boundaries between them. Each formal world can be described as a type of validity claim, detailed in the left sidebar.
The grounds for a subjective claim are based in an individual's experience, which each of us have alone. While an individual has privileged access to their experiences, they do not have direct access, as any of these claims are interpreted by them in the process of realizing the claims exist; when they have awareness of them. The subject can be wrong about what they are experiencing, and can be reproduced in a systemic way through psychological processes like denial. Normativeevaluative claims must be rational, in the sense that they are internally coherent, and are not merely a matter of opinion; these claims should have an implied argumentative structure built around them, which indicate consensus based on reasons. In the expression of communicative acts, the actor "demarcates" their own unique position in relation to all three of the formal worlds; no single world contains the identity claim that might be said to constitute the "I" component.

\section{Intersubjective Space}

For any given communicative act, we as actors have a felt experience, and fused within that experience are all three subject positions: 1 ) the subjective or limited access, individual sense of the "I"; 2 ) the normative perspective of what ought to be; and 3) the objective or multiple access perspective, or the individual and collectivist sense of "me." Only as these three validity claims first fuse and then emerge together does our act make sense. Through this reciprocal and reflexive process of acting and forming validity claims surrounding that act, an intersubjective space becomes possible between two individuals and mutual understanding is able to emerge. This intersubjective space forms whenever we act communicatively. By acting in a communicative space, we have already taken on multiple subject positions, and the validity of 
our acts involving meaning are assumed. It is possible, however, to consciously determine the validity of our acts, either during or just after we act, deliberately taking on other subject positions to assess the validity of our heretofore implicit validity claims. When we, even as solitary actors, position-take in this way, we imagine how our communicative acts might be viewed by others, either regarding the meaning of an act or the next possible act(s), which is inherently intersubjective. "It is through norms, 'rules,' that position-taking is made possible. They are the hinges upon which we swing from first to second and third person positions. It is only because of norms that position-taking is at all possible" [7, p. 1024]. It is also important to note the role of reflection, as it is understood in the design discourse (i.e., Schön's "reflection-in-action," [20]) in relation to this concept of position-taking: "Position-taking is what structures higher levels of thought...It is the basis of what we mean by 'reflection.' Thinking is dialoging internally Reflecting is taking a second and/or third person position in relation to one's own thoughts." [7, p. 1023]. This use of reflexivity is at the core of being ethical and value-centered as a designer [21,22], and thus it is vital for researchers to understand how to actively move between subject positions.

\section{Integrated Methods to Support Meaning \\ Reconstruction}

Within the epistemological and ontological grounding of the previous section, Carspecken $[6,7]$ has proposed a set of integrated methods to deeply investigate communicative acts. These methods are often described as supporting meaning reconstruction-a rigorous process through which the researcher builds an understanding of the potential range of meaning and validity claims that are inferred by a communicative act. The process of meaning reconstruction builds upon speech act theory, TCA, and Giddens' structuration in multiple ways, resulting in a toolkit that allows the researcher to deeply analyze communicative acts, a first step towards understanding power relations, normative implications, and the ways in which communication impacts and reproduces societal norms.

We will introduce two specific methods that allow the researcher to engage in meaning reconstruction: meaning fields and validity horizons. These methods draw on the vocabulary presented above, and contain some of the intermediary steps needed to build a rich understanding of communicative acts collected through physical or digital fieldwork. These methods serve as a "bridge" to developing an understanding of structures that underlie the system(s) being analyzed, allowing for a fuller conversation of system relations on the domainspecific level and beyond.

\section{Meaning Fields}

In generating a meaning field, the goal is to explore possible meanings for a given communicative act"meanings that other people in the setting might themselves infer, either overtly or tacitly" [6, p. 95]. In this process, a bounded set of possible meanings for the given communicative act can be explored, not to determine the "true" meaning intended by the actor, but rather a paradigmatic set of meaning possibilities. The resulting meaning fields may not be an exact articulation of a field that the actor themself might generate, recognizing that, in an analytic sense, "meanings are always experienced as possibilities within a field of other possibilities" [6, p. 96], and that 
actors may be largely unaware of portions of their meaning field.

Resulting meaning fields are a generative step in the reconstructive analysis process, identifying clusters of potential meanings, and their relationship to one another. Clusters are commonly related through logical "and," "or," "and/or," and "or/and" statements to demonstrate potential pairings of potential meanings, which can be more fully explored in the working out of a validity horizon. This method is ideally suited to explore meaning-making beyond what can be directly observed or self-reported by participants. Meaning fields are used to explore ranges of potential meanings from several different perspectives, surfacing portions of conversations where deep, tacit meanings are frequently unstated yet important to the overall communicative process.

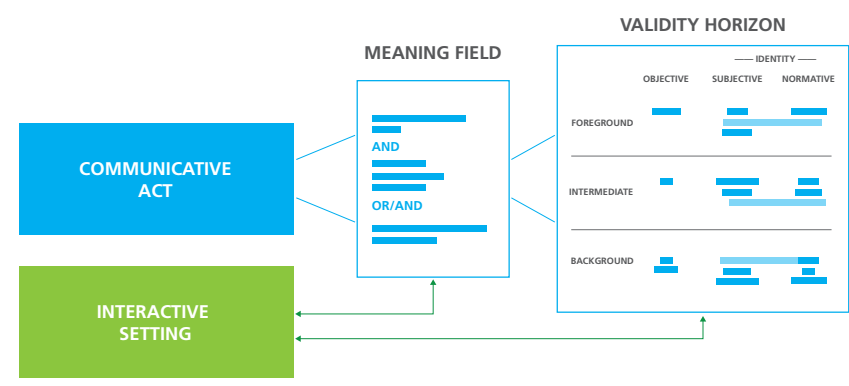

Figure 1. Relationship of methodological concepts in the analysis of a communicative act.

\section{Validity Horizons}

Validity horizons build on the work done in creating a meaning field, exploring what validity claims from each of the three formal worlds must be instantiated in tacit or explicit form for the given meaning to make sense. Figure 1 visually depicts the interaction between analysis of a specific communicative act and the generation of a meaning field and validity horizon. This figure also underscores the generative nature of these methods in informing changes to understandings of the setting infrastructure, including the ways in which actors take on roles and typifications and operate within specific fields of content coherence. Zhang and Carspecken [24] provide a fuller explanation of the purpose of this method:

"The process of articulating 'validity horizons' in qualitative data analysis [6] involves moving inferentially connected portions of the background of a meaning horizon into explicit articulation. [...] the structures that are instantiated by meaningful acts have inferential implications that exceed even the tacit awareness of an actor. [...] They can also be noticed and articulated by an outside observer in ways that none of the actual participants have any awareness of (if the observer takes a performative position, i.e., is a virtual participant)." [p. 209]

In reconstructing the inferential implications of a given meaning, validity claims are produced-following the categories of objective, subjective, and normative, discussed above. Another special category of validity claim, an identity claim, may also be mapped in the validity horizon, which is a combination of a subjective and normative claim-something that is implicitly claimed as part of the identity of the person in a subjectively normative sense, representing one's personal involvement in reproducing and shaping a social context $[6,7]$. All validity claims are then distributed along a continuum from highly foregrounded 
(i.e., likely to be immediately salient to the actors) to highly backgrounded (i.e., answers to the most baseline "why" questions). These highly backgrounded claims are often outside of the scope of what the actors themselves might even be aware of, and they are especially useful in understanding the ethics of the underlying social system that is being perpetuated.

\section{Two Case Examples}

To demonstrate this critical qualitative approach, we will describe two communicative acts that relate to issues of inclusivity in hacking communities, using meaning fields and validity horizon analysis to explicate the validity claims that are inferred by the acts.

Example 1: Diversity in a Midwestern Hackerspace In an annual meeting of a small Midwestern hackerspace, a board member raised the issue of membership, noting the community was experiencing an uncharacteristic dip in numbers. It is important to note, here that this "dip" was only 3-4 members, but as this hackerspace had an average membership of around 25 at that time, this was characterized as more serious. The people who were on the membership roster but were in the process of leaving-which included the only female member at the space who was not also a member of the board-were discussed, and one member asked if any of them explained why they were leaving. Several potential explanations were put forth, with the consensus being that many of them did not have the time to participate. However, there was also the sense that that there might also be a deeper explanation for them leaving. Another board member, now the only female member of the hackerspace, said, "If there's something we can fix that drove them away, then we should worry about it. But otherwise there are lots of reasons people might stop coming. As long as we make sure that we are all excellent to each other then that's the best that we can do." The meaning fields in the sidebar and the validity horizon in Table 1 were constructed based on this quote.

Reading through the meaning field, it might appear that some of the potential meanings presented are speculations on the part of the researcher because they are not clearly visible in the quote itself. However, this is not mere speculation, as the potential meanings present in the meaning field are built on the researcher's previous interactions with the participant in question, and are supported through field notes, researcher notes, reflective journals, and other such tools. Therefore, the validity of this method is dependent on long-term engagement, making it less speculative-but rather an informed deconstruction of 1) what the actor could possibly be intending, and 2) the multiple ways that the actor's interlocutors could be interpreting the actor's speech act. In this particular case, it is relevant to note that during previous such conversations about how the space could expand its membership to include more women, this board member had consistently expressed her opinion that it was not worth attempting to alter their behavior to attract more women because she felt comfortable in the space, so other women should also not have a problem feeling comfortable there. Based on those previous interactions, the meaning field includes subtle references to previous discussions, of which other interlocutors present would have been aware.

In the next stage, constructing the validity horizon, claims within the meaning field are organized based on the three formal worlds described above, as well as the 


\section{Example 1 Meaning Field}

"If they didn't say anything" THEN "we can't have done anything wrong" AND "our current behavior is perfectly acceptable for anyone" BECAUSE "it is acceptable for us" AND "we should treat everyone exactly the way we already treat each other"

OR/AND "Hackerspaces are not for everyone" AND "we should not feel bad if someone just does not fit in"

OR/AND "we lost one of our few female members" BUT "I do not think that it was our fault" AND "I am female" AND "I feel comfortable here" THEREFORE "we are not doing anything that discourages female participation" EVEN THOUGH "there are no other females here"

OR/AND "women should be treated exactly the same as men" AND "the policy here is that everyone be 'excellent to each other'" AND "that is a reasonable policy for anyone" AND "women should not be given special treatment or attention"

\begin{tabular}{|c|c|c|c|c|}
\hline & OBJECTIVE & SUBJECTIVE & NORMATIVE & IDENTITY \\
\hline \multirow{2}{*}{ 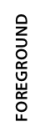 } & \multirow{2}{*}{$\begin{array}{l}\text { We lost a few members. } \\
\text { It was not our fault / "shit } \\
\text { happens." }\end{array}$} & \multirow[t]{2}{*}{ I feel comfortable here. } & $\begin{array}{l}\text { We should not be worried about } \\
\text { our behavior. }\end{array}$ & \multirow[t]{2}{*}{$\begin{array}{l}\text { I am/we are good and } \\
\text { reasonable people. }\end{array}$} \\
\hline & & & $\begin{array}{l}\text { We should not try to change } \\
\text { anything about our community. }\end{array}$ & \\
\hline \multirow{3}{*}{ 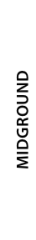 } & \multirow[t]{3}{*}{$\begin{array}{l}\text { One of the members we lost } \\
\text { was one of very few female } \\
\text { members. }\end{array}$} & \multirow[t]{3}{*}{$\begin{array}{l}\text { I'm worried we did something } \\
\text { to push them away. }\end{array}$} & $\begin{array}{l}\text { "Be excellent to each other" is } \\
\text { a sufficient guiding philosophy } \\
\text { for our community. }\end{array}$ & $\begin{array}{l}\text { I can speak for other women's } \\
\text { experiences. }\end{array}$ \\
\hline & & & $\begin{array}{l}\text { Everyone should feel } \\
\text { comfortable here. }\end{array}$ & \multirow{2}{*}{$\begin{array}{l}\text { I am an authority figure in } \\
\text { this space. }\end{array}$} \\
\hline & & & $\begin{array}{l}\text { Everyone should be treated } \\
\text { exactly the same. }\end{array}$ & \\
\hline \multirow{2}{*}{ 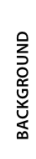 } & \multirow[t]{2}{*}{ Some people just don't fit in } & \multirow[t]{2}{*}{$\begin{array}{l}\text { I don't want it to have been } \\
\text { our fault }\end{array}$} & $\begin{array}{l}\text { We should ignore people's } \\
\text { backgrounds and personal } \\
\text { histories. }\end{array}$ & \multirow[t]{2}{*}{ I am comforting the group. } \\
\hline & & & $\begin{array}{l}\text { We should not try to cater to } \\
\text { everyone. }\end{array}$ & \\
\hline
\end{tabular}

Table 1. Validity horizon documenting validity claims for Example 1 by formal world.

identity claims that come from a combination of subjective and normative validity claims. It would be more typical to include just one section of the meaning fields at a time in a validity horizon, but for the sake of brevity, while also demonstrating the strength of this method in uncovering tacit norms expressed through

communicative acts, we have incorporated a composite of each meaning field into the validity horizon.

The foreground and mid-ground objective claims of this speech act found in Table 1 are straightforward to reconstruct, as they are the subject of the act: that several people have left the community and that one of them was one of the few female members of that space. Though she was not highlighted in this members quote any more than the other former members, it was clear based on the nature of the discussion and the motivation behind even offering a justification for the vanishing members that her leaving could require a reevaluation of the space's policies were it to be found that the space was not as welcoming to women as it was once thought. The subjective claims in this validity horizon are rooted in the well-documented empirical observations of the researcher across time, as well as a thoughtful interpretation of the motivations for her attempting to justify the vanishing members, as well as how that motivation intersected with that actor's role in the space as an authority figure.

This validity horizon for this quote appears to be heavily normative. When this happens, it is important to consider whether or not that matches what would be expected, given the context of the given speech act. In this case, it is appropriate because this comment was made during a part of the discussion in which members were discussing what, if anything, should be done about the problem at hand. The normative claims for this particular quote are broken down into what should be done to solve the problem (which resulted in an abdication of responsibility: "it was not our fault so we can safely ignore any implication we might have"), as well as claims for how others should be experiencing 
the space. In this instance, the tension between the normative claim that others should also feel comfortable in the space and the claim that the community should already be comfortable enough for everyone provides a communicative space to explore how members of this community might feel they do or do not live up to these expectations.

Reconstructing the identity claims in this validity horizon relied on interpreting the rhetorical function of the quote more so than interpreting the quote itself.

The particular temporal location of the communicative act within the conversation marked a moment where the conversation could have either split into a critical discussion of the group's failings, or a supportive and comforting discussion that assuaged the group's fears about what they may or may not be doing incorrectly. Because this actor chose the comforting route, and since going the comforting route is typical for this particular actor in this space, it is clear that she was further enacting previously expressed identity claims of being the "nurturer" or "comforter" in this space.

\section{Example 2: Listserv Discussions}

The second example comes from a lengthy discussion on the hackerspaces.org Discuss listserv about safe space policies, including anti-sexual-harassment policies, and how and why they should or should not be implemented in hackerspaces. This discussion was one of the top ten longest discussion threads ever to take place on this listserv as of July 2015. The quote analyzed in this second example comes from the most prolific poster in the listserv, who proposed that hackerspaces should not attempt to implement such policies because they risk alienating or "artificially" altering the currently-existing community in that space. A brief portion of the overall post is included below

"I think this whole discussion hinges on a very simple question. Why is it worthwhile to artificially promote a change in an existing community. If the answer is because the hackerspace should be inclusive to everyone, my answer is no, it should not be. By it's very nature it's already exclusionary. It's a hackerspace. Not a bake shop. Not a petting zoo. Not a race track. It has a specific focus, and by that it is already exclusionary. More to the point, hackerspaces are built around communities. And communities themselves are exclusionary. If you don't jive well with a community, you don't belong to that community, go find another one. If you think that your hackerspace can be home to all the peoples, you aren't building a hackerspace you are building a public library, and by all means enjoy the crackheads and good luck keeping that inclusive to everyone. Ask noisebridge how that went for them." $[\ldots]$

As with the previous example, the construction of this meaning field relies on the researcher's extended presence in this community, including knowledge of this particular author. In constructing the meaning field that appears to the left, each line of the quote is analyzed and expanded to consider potential meanings that were either intended by the author or potential meanings that could have been interpreted by others reading the email and participating in the discussion. With each of these expansions, further clarification is added in the form of backgrounded claims that would need to be true for the potential meaning to be consistent or make sense. These background claims also appear in the 


\section{Example 2 Meaning Field}

"The existing community should take precedent over any subsequent community" AND "anything that changes that community from the outside is artificial/bad" BECAUSE "that community already works/is good" AND "should be allowed to change naturally." AND "It is more important to cater to who fits in already than to consider who could fit in."

\section{OR / AND}

"Hackerspaces should not try to be inclusive" BECAUSE "trying to be broadly inclusive will lead to 'undesirables' like Noisebridge" AND "Noisebridge is a bad model" AND "hackers can't handle inclusivity and diversity" BECAUSE "hackers are strange/odd/unwell" AND "a

hackerspace is not a public facility" AND THEREFORE "it does not have to cater to everyone"

\section{OR / AND}

"It is not important to worry about who is or is not included in the space" BECAUSE "people either fit in or not on their own" AND "People who don't feel like they fit in must not really share our interests" OTHERWISE "they would feel comfortable" AND "people should be able to adapt to hostile environments if they are interested."

\begin{tabular}{|c|c|c|c|c|}
\hline & OBJECTIVE & SUBJECTIVE & NORMATIVE & IDENTITY \\
\hline \multirow{2}{*}{ 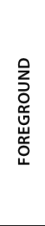 } & \multirow[t]{2}{*}{$\begin{array}{l}\text { Hackerspaces are communities } \\
\text { that are NOT public spaces. }\end{array}$} & $\begin{array}{l}\text { I worry that being inclusive to } \\
\text { everyone will lead to including } \\
\text { people who are undesirable } \\
\text { (e.g., crackheads). }\end{array}$ & $\begin{array}{l}\text { Existing communities and their } \\
\text { underlying culture should be } \\
\text { protected against "artificial" } \\
\text { change. }\end{array}$ & \multirow{2}{*}{$\begin{array}{l}\text { I am an authority on this issue } \\
\text { I am not the type of person to } \\
\text { hedge or not tell things the } \\
\text { way that they are. }\end{array}$} \\
\hline & & $\begin{array}{l}\text { I would not want to end up like } \\
\text { Noisebridge, whose problems } \\
\text { include being over-inclusive. }\end{array}$ & $\begin{array}{l}\text { "Artificial changes" should be } \\
\text { discouraged. }\end{array}$ & \\
\hline \multirow{3}{*}{ 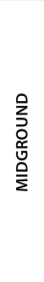 } & $\begin{array}{l}\text { This is a controversial issue } \\
\text { with multiple sides. }\end{array}$ & \multirow[t]{3}{*}{$\begin{array}{l}\text { I am frustrated that this conver- } \\
\text { sation is taking place. }\end{array}$} & $\begin{array}{l}\text { If participants are truly } \\
\text { "hackers," they will share our }\end{array}$ & \multirow[t]{3}{*}{$\begin{array}{l}\text { I understand what is good for } \\
\text { hackerspaces and what is not. }\end{array}$} \\
\hline & \multirow[t]{2}{*}{$\begin{array}{l}\text { Hackers are not "normal" and } \\
\text { are exactly the wrong people } \\
\text { to understand complex social } \\
\text { issues. }\end{array}$} & & $\begin{array}{l}\text { interests and should feel com- } \\
\text { fortable here. Hackerspaces } \\
\text { should not worry about being } \\
\text { inclusive. }\end{array}$ & \\
\hline & & & $\begin{array}{l}\text { Hackers should focus on the } \\
\text { people who already fit in, rath- } \\
\text { er than those who could fit in. }\end{array}$ & \\
\hline \multirow{2}{*}{ 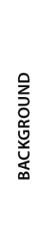 } & \multirow{2}{*}{$\begin{array}{l}\text { The community that already } \\
\text { exists in a given space should } \\
\text { only change on its own. } \\
\text { The existing community is } \\
\text { already functioning, and thus } \\
\text { "perfect" in some sense. }\end{array}$} & \multirow[t]{2}{*}{$\begin{array}{l}\text { I feel defensive when I think my } \\
\text { community's culture might be } \\
\text { under attack. }\end{array}$} & $\begin{array}{l}\text { We should be careful and } \\
\text { serious about policy changes } \\
\text { that alter the culture of the }\end{array}$ & \multirow[t]{2}{*}{$\begin{array}{l}\text { I wish to keep hackerspaces } \\
\text { "pure" in the ways I deem } \\
\text { appropriate. }\end{array}$} \\
\hline & & & $\begin{array}{l}\text { People should be able to } \\
\text { adapt to or overcome hostile } \\
\text { environments if their interests } \\
\text { are aligned enough. }\end{array}$ & \\
\hline
\end{tabular}

validity horizon. One section of the meaning field depended on various interpretations of the actor's use of the word "artificial" in his email, as well as the clear connotation he was implying that artificial change was undesirable. Intended and interpreted connotations, in general, are good places to start investigating more deeply into backgrounded claims.

The process of expanding claims from the meaning field to a validity horizon (Table 2 ) uncovered several tacit normative assumptions that would have been difficult to discuss otherwise. Particularly interesting in this case is the tension that exists between discouraging

"external" or "artificial" change while also attempting to leave room for the community to be able to grow on its own, without defining the difference between what would be considered "artificial" versus "natural" change. In what way, exactly, is a proposal originating from within the community for implementing a safe space policy "artificial" or "external"? At first glance this actor's criticism of external policies might seem reasonable, as he is playing off of the primacy of the already-established group. But once the distinction here is investigated, it falls apart. Similarly, the assumption that "people should be able to adapt to or overcome hostile environments if their interests are aligned enough" is particularly illuminating. This actor appears to assume that the set of people who are interested in hacking but do not feel comfortable in hackerspaces does not exist or is not important, as he dichotomizes the potential population into either people who are interested in hacking and fit in, or people who do not fit in because they are not interested in hacking.

Table 2. Validity horizon documenting validity claims for Example 2 by formal world. 


\section{Discussion}

We have demonstrated how working through this particular set of integrated qualitative research methods highlights critical dimensions of communication, explored in a reflexive way between the collected data and the analysis of that same data by the researcher. This is not a clinical interpretation or reading of a critical design in the mode of a 'critic,' but rather relies on the researcher's long-term engagement with the actors in question as well as the actor's interlocutors in order to reconstruct both what the speech act was intended to convey as well as how that speech act could have reasonably been interpreted by others. In many of the cases in which this method may be employed, the researcher is not an external, fly-onthe-wall observer, but a co-participant; one who the participants do not just see as an "objective" resource but as a bona fide member of their community.

A key characteristic of this analysis is its transparency, demonstrating with multiple interlinked methods the mental rigor that is required on the part of the researcher to connect multiple communicative acts with the potential social phenomena they reproduce or otherwise contribute to. While the transparency of method is itself a valuable reflective exercise for the researcher, the methods also leave an "audit trail" that is useful in demonstrating the rigor in thinking through what validity claims may be being made at multiple levels of awareness and validity category. A sustained interest in increased methodological transparency also raises the question of what can or should constitute rigor in $\mathrm{HCI}$ research. The approach we suggest here is only one of many that could build rigor in the research process, opening this question for further scrutiny.
It would not be typical to perform a full meaning field and validity horizon on a large number of speech acts in a given data source-there simply would not be enough time. Rather, the strength of this method comes through applying it to particularly salient quotes-such as the ones above, which happen to be uncharacteristically normative in nature already-to more closely investigate themes that have been seen throughout the data. In addition, this method can be used throughout a period of long-term to investigate hypotheses regarding normative commitments, or to engage in "strip analysis" [1]-where emergent hypotheses lead to additional analytic and fieldwork activities to validate or exclude such hypotheses.

\section{Conclusion}

In this paper, we have introduced a set of critical qualitative methods and documented their utility in highlighting areas of normative concern that are of interest to the $\mathrm{HCI}$ community. Our intent is not to supplant the variety of standpoints represented by critical research, but rather to encourage this research to be conducted in highly rigorous ways. Meaning reconstruction is one such grouping of methods that allows the researcher to engage in a reflexive way with her data, demonstrating findings that intersect with criticality in a variety of ways while holding high methodological standards in the qualitative tradition.

\section{Acknowledgements}

This research was funded in part by NSF IIS Creative IT (\#1002772) and the Intel Science and Technology Center for Social Computing. 


\section{References}

1. Michael Agar. 1986. Speaking of ethnography. Sage Publications, Beverly Hills, CA.

2. Shaowen Bardzell, and Jeffrey Bardzell. 2011. Towards a feminist HCI methodology: social science, feminism, and HCI. In Proceedings of the SIGCHI Conference on Human Factors in Computing Systems, 675-684.

3. Jeffrey Bardzell, and Shaowen Bardzell. 2015. Humanistic HCI. Morgan Claypool Publishers.

4. Jeffrey Bardzell, Shaowen Bardzell, and Lone K. Hansen. 2015. Immodest Proposals: Research Through Design and Knowledge. In Proceedings of the SIGCHI Conference on Human Factors in Computing Systems.

5. Jeffrey Bardzell, Shaowen Bardzell, and Erik Stolterman. 2014. Reading critical designs: supporting reasoned interpretations of critical design. In Proceedings of the SIGCHI Conference on Human Factors in Computing Systems, 19511960.

6. Phil F. Carspecken. 1996. Critical ethnography in educational research: A theoretical and practical guide. Routledge, New York.

7. Phil F. Carspecken. 2003. Ocularcentrism, phonocentrism and the counter enlightenment problematic: Clarifying contested terrain in our schools of education. The Teachers College Record 105, 6: 978-1047.

8. Phil F. Carspecken, and Laurie MacGillivray. 1998. Raising Consciousness about Reflection, Validity, and Meaning. In Being Reflexive in Critical Educational and Social Research, Geoffrey Shacklock and John Smyth (editors). Falmer Press, London, UK, 171-190.

9. Barbara Dennis, Lucinda Carspecken, and Phil Carspecken (Eds.). 2013. Qualitative Research: A Reader in Philosophy, Core Concepts, and Practice. Peter Lang Publishing, New York, NY.
10. Steve Harrison, Deborah Tatar, \& Phoebe Sengers, 2007. The three paradigms of HCI. In CHI EA: CHI'07 Extended Abstracts on Human Factors in Computing Systems.

11. Yvonna S. Lincoln and Egon G. Guba. 1985. Naturalistic inquiry. Sage Publications, Beverly Hills, CA.

12. Carl DiSalvo. 2012. Adversarial design. MIT Press, Cambridge, MA

13. Paul Dourish, Janet Finlay, Phoebe Sengers, and Peter Wright. 2004. Reflective HCI: Towards a Critical Technical Practice. In Proceedings of the SIGCHI Conference on Human Factors in Computing Systems, 1727-1728.

14. Colin M. Gray. 2014. Living in two worlds: A critica ethnography of academic and proto-professional interactions in a human-computer interaction design studio. PhD dissertation, Indiana University, Bloomington, IN.

15. Colin M. Gray, and Craig D. Howard. 2015. Normative Concerns, Avoided: Instructiona Barriers in Designing for Social Change. In Analyzing Design Review Conversations, Robin S. Adams, Patrice Buzzanell and Junaid A. Siddiqui (editors). Purdue University Press, West Lafayette, IN, 241-260.

16. Steve Harrison, Phoebe Sengers, and Deborah Tatar. 2011. Making epistemological trouble: Thirdparadigm $\mathrm{HCI}$ as successor science. Interacting with Computers 23, 5: 385-392. http://doi.org/10.1016/j.intcom.2011.03.005

17. Joe L. Kincheloe, and Peter McLaren. 2011. Rethinking critical theory and qualitative research. In Key Works in Critical Pedagogy, k. hayes et al. (editors). Sense Publishers, 285-326.

18. James Pierce, Phoebe Sengers, Tad Hirsch, Tom Jenkins, William Gaver, and Carl DiSalvo. 2015. Expanding and refining design and criticality in HCI. In Proceedings of the 33rd Annual ACM Conference 
on Human Factors in Computing Systems, 20832092. http://dx.doi.org/10.1145/2702123.2702438

19. Steve Sawyer, and Mohammad H. Jarrahi. 2014. Sociotechnical approaches to the study of information systems. In A. Tucker and $\mathrm{H}$. Top (editors) CRC handbook of computing. Chapman and Hall, New York, NY.

20. Donald A. Schön. 1983. The reflective practitioner: How professionals think in action. Basic Books, New York, NY.

21. Phoebe Sengers, Kirsten Boehner, Shay David, and Joseph 'Jofish' Kaye. 2005. Reflective design. In CC'05: Proceedings of the 4th decennial conference on Critical computing: between sense and sensibility, 49-58.

22. Katie Shilton. 2012. Values levers: Building ethics into design. Science, Technology \& Human Values
38, 3: 374-397.

http://doi.org/10.1177/0162243912436985

23. Austin L. Toombs, Shaowen Bardzell, and Jeffrey Bardzell. 2015. The Proper Care and Feeding of

Hackerspaces: Care Ethics and Cultures of Making. In Proceedings of the 33rd Annual ACM Conference on Human Factors in Computing Systems, 629638.

24. Ran Zhang, and Phil Carspecken. 2013. Content inference fields in intersubjective space:

Transpersonal position, illocution, and logic in the analysis of human interactions. In Qualitative

Research: A Reader in Philosophy, Core Concepts, and Practice, Barbara Dennis, Lucinda Carspecken and Phil F Carspecken (editors). Peter Lang Publishing, New York, NY, 201-242. 


\section{Commentary}

For alt.chi Paper

Meaning Reconstruction as an Approach to Analyze Critical

Dimensions of HCI Research

\section{Ellie Harmon}

Department of Information Science University of Colorado Boulder ellie@ellieharmon.com
In motivating their new-to-HCI method, the authors argue that contemporary HCI - "marked by criticality, sociality, and power" - lacks sufficiently rigorous methods. In contrast to first and second paradigm HCI, which "operated in an experimental scientific mode, using measures that were easily quantified and compared," third paradigm methods "have been more diffuse, drawing on numerous social science and humanistic traditions." Such work "is often a 'black box,' lacking clear description of how critical insights are drawn from data in a rigorous way." Yet, it seems to me that much HCI research - regardless of criticality - often appears on the scene in the same symptomatic "black box." Yet, criteria for evaluating "contributions" generally hinge on the performance of novelty - a new system, design, or insight - not the performance of 'rigor,' good science, or good research; methods are allotted a minimal amount of space in papers - in favor of featuring novel results. Meanwhile, alt.chi - a zone on the fringes of the discipline - is not an uncommon venue for methods papers. Is an apparent lack of rigor a problem specific to third paradigm $\mathrm{HCI}$, or symptomatic of a general lack of care about methods in the first place? And, what does rigor mean, anyway?

Notably, the explication of a rigorous method in this paper appears to transform social discourse into something that looks like discursive data. The analysis appears quite traditionally empiricist: it has a set of clear steps, and even involves the production of diagrams and tables. Is this what it means to be rigorous? Is rigor the result of a specific practice, following 'properly' a set of steps? Must it result in the data-fication of evidence? Can a more multiple and diffuse approach not also be rigorous?
When starting my PhD, I took a series of classes in the anthropology department, hoping to learn how the real ethnographers did fieldwork. Disappointingly, at the time, I found few methodological prescriptions. Rather, I would be referred to such illuminatingly clear explications of method as Hugh Gusterson's essay on "polymorphous engagement," which says, roughly: 'Go out and talk to some people! Can't get access to the place where you should do participant observation? No big deal, go somewhere else!

Socialize! Read the newspaper! Think about it. Write." Such an anthropology can appear free-wheeling - to say the least - and yet, I also learned that not just anything goes. Method and rigor still mattered.

For myself, I have come to think of rigor as bound up in the orientation and commitment with which one approaches the craft of spinning a story out of a set of empirical evidences; more personal and political than stepwise or scientific; involving a vulnerability to research participants, aspirations to tell truths that resonate with local understandings, and attention to one's location within the power structures of research, writing, and authority-making. Thus, while I still find much "ethnographic" work within $\mathrm{CHI}$ to appear quite shallow and less-than-thick, I am also skeptical that specifying a regimented method (be it meaning reconstruction or a form of the perennial favorite, grounded theory) will help. Thus, while I value the introduction of meaning reconstruction and can see its usefulness for my own and others' work, I'm less certain that it addresses the problem of rigor that the authors raise. However, this question of rigor - what constitutes it, how to assess it, why it might be important - seems a crucial issue for the field to discuss, and not just on its alt/fringes. 